\title{
Drawing Database Schemas with DBdraw
}

\author{
Giuseppe Di Battista ${ }^{1}$, Walter Didimo ${ }^{2}$, Maurizio Patrignani ${ }^{1}$, and \\ Maurizio Pizzonia ${ }^{1}$ \\ 1 Dipartimento di Informatica e Automazione, Università di Roma Tre, Via della \\ Vasca Navale 79, 00146 Roma, Italy. \{gdb, patrigna,pizzonia\}@dia.uniroma3.it \\ 2 Dipartimento di Ingegneria Elettronica e dell'Informazione, Università di Perugia, \\ Via G. Duranti 93, 06125 Roma, Italy. didimo@diei.unipg.it
}

\section{Short Description}

DBdraw is an application that allows the user to automatically produce drawings of database schemas according to a drawing standard that is well accepted by the database community. The drawing engine of DBdraw is based on the GDToolkit library.

\section{Areas of Application}

DBdraw is clearly targeted to developers and maintainers of databases. It may give a valuable help in building first release documentation as well as in facing the lack of documentation of old systems.

\section{Layout: Algorithms and Features}

The drawing standard adopted by DBdraw represents the tables of the schema with boxes, and table attributes with distinct stripes inside each table. Links connecting attributes of two different tables represent referential constraints or join relationships, and may attach arbitrarily to the left or to the right side of the stripes representing the attributes. The drawing algorithm is inspired by the topology-shape-metric approach. Briefly, it consists of four steps:

Constrained Planarization. A planarization is performed on the graph underlying the database schema in order to obtain a planar embedding consistent with the specific sequence of attributes of the table. Dummy vertices of degree four are introduced to replace crossings, see Figure 1(a)

U-Turns Assignment. In our drawing convention each link may monotonically follow in the left-to-right direction or may perform one or more u-turns. In this step a (possibly empty) sequence of u-turns is associated with each link trying to minimize their total number, see Figure $1(\mathrm{~b})$

Orthogonalization. This step associates an orthogonal shape to the schema. Each u-turn is replaced with two bends of 90 degrees. The shape is such that links approach tables horizontally, see Figure 1(c)

Constrained Compaction. The output of this step is a complete drawing of the database schema. The length of the link and the size of the vertices are 


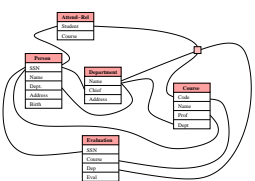

(a) Constrained planarization

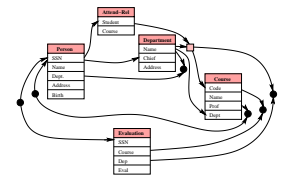

(b) U-turn assignment

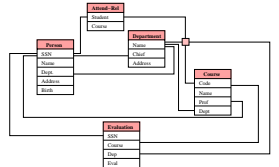

(c) Orthogonalization

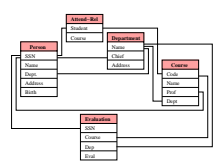

(d) Constrained compaction

Fig. 1. Illustration of the main steps of the DBdraw algorithm.

computed, keeping as small as possible the area and the total link length. The adopted technique allows us to exactly specify the incidence point of each link on the tables involved in the link. Dummy vertices introduced in the Constrained Planarization step are removed, see Figure 1(d).

\section{Architecture and Interfaces}

The architecture of DBdraw takes advantage of the capability provided by the Windows ${ }^{\circledR}$ operating system for importing database schemas and producing an output that may be rapidly inserted into database documentation. The user specifies a database, DBdraw extracts the schema from the database, computes the drawing and inserts it in a Microsoft ${ }^{\circledR}$ Word document whose name is again specified by the user. The drawing is stored in a vectorial format and may be further modified. Currently only Microsoft ${ }^{\circledR}$ Access databases (.mdb files) are supported. The drawing engine is written in $\mathrm{C}++$ and it is based on the GDToolkit graph drawing library. The other parts of the system are written in Visual Basic.

\section{Screenshots}
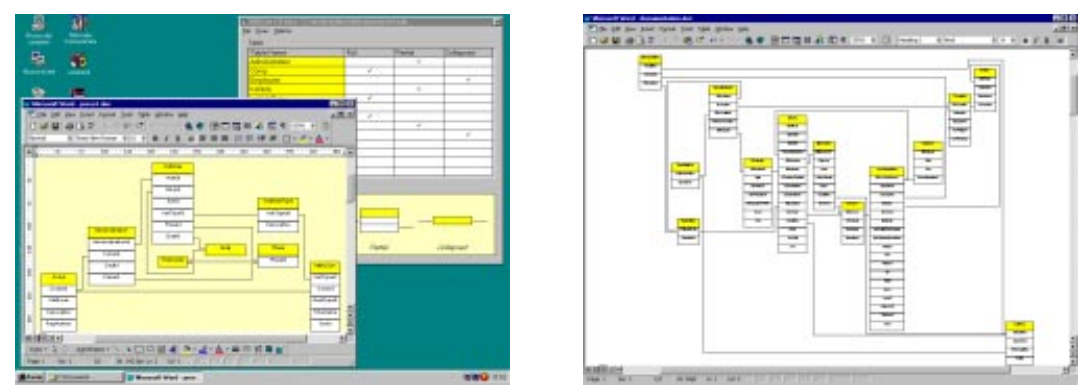

\section{References}

1. G. Di Battista, W. Didimo, M. Patrignani, and M. Pizzonia. Drawing relational schemas. In W. C. de Leeuw and R. van Liere, editors, Data Visualization 2000, Eurographics. Springer-Verlag Wien, 2000. 